\title{
Calidad de vida y problemas bucales en preescolares de la provincia de Huaura, Lima.
}

\author{
Quality of life and oral problems in the province of preschool Huaura, Lima.
}

Roxana Patricia López-Ramos ${ }^{1, a}$, Carmen Rosa García-RupayaC ${ }^{1, b}$.

\section{RESUMEN}

Objetivos: Evaluar el impacto de los problemas bucales en la calidad de vida en niños de 3 a 5 años de la Institución Educativa Inicial del distrito Hualmay de la provincia de Huaura, departamento de Lima en el 2011. Material y Métodos: Este estudio fue de tipo descriptiva, transversal. Evaluó a 153 niños, a quienes se les examinó la cavidad bucal y al responsable del cuidado del niño se aplicó el Early Childhood Oral Health Impact Scale (ECOHIS); previamente se realizó la validación peruana. Un examinador calibrado evaluó tres problemas bucales: caries de infancia temprana, traumatismos dento-alveolares y maloclusiones en dientes anteriores. Se realizó el análisis estadístico; aplicando pruebas de Anova, U Mann Whitney y Kruskal Wallis con un nivel de significancia de 95\%. Resultados: La frecuencia de caries de infancia temprana fue de $76,47 \%$; de traumatismos dento-alveolares $9,8 \%$ y de maloclusiones anteriores $6,54 \%$. Las preguntas mayormente respondidas del ECOHIS fueron sobre: el dolor (54,9\%), dificultad para comer $(49,7 \%)$ y beber $(41,9 \%)$. La dimensión limitación funcional del ECOHIS presentó el promedio más alto $(4,75 \pm 3,44)$. La severidad alta de caries de infancia temprana obtuvo el promedio más elevado en relación al ECOHIS $(24,88 \pm 9,43)$. Conclusiones: La caries de infancia temprana presentó impacto negativo en la calidad de vida relacionada a la salud bucal de los niños evaluados.

\section{Palabras Clave: CALIDAD DE VIDA, SALUD BUCAL, PREESCOLAR.}

\begin{abstract}
\footnotetext{
Facultad en Estomatología. Universidad Peruana Cayetano Heredia. Lima, Perú.

a Residente Especialidad Odontología Pediátrica,

b Docente del Programa de Maestría en Estomatología.
}

Objective: To assess the impact of oral problems on quality of life related to oral health children (OHRQoL) of 3-5 years old of an initial school of a district of the Huaura province, Lima department in 2011. Methods: This research was descriptive, cross, evaluated 153 children who were examined their oral cavity and applied to caregiver the Early Childhood Oral health Impact Scale (ECOHIS). Before was validated the Peruvian version. A examiner calibrated evaluated three oral problems: early childhood caries, injuries dento-alveolar and above malocclusions. Stadistics analysis; was performed, 
used tests as: Anova, U Mann Whitney and Kruskal-Wallis. Results: The frequency of early childhood caries was 76,47\%, of injuries dento-alveolar 9,80\%, above malocclusions $6,54 \%$. The questions most often answered of the ECOHIS were related: pain $(54,9 \%)$, difficulty eating $(49,7 \%)$ and drinking $(41,9 \%)$. The functional dimension ECOHIS presented the highest mean (4.75 \pm 3.44$)$. The high severity of early childhood caries on ECOHIS presented the highest average $(24.88 \pm 9.43)$. Conclusion: Early childhood caries presented negative impact on oral health-related quality of life related (OHRQoL).

Key Words: QUALITY OF LIFE, ORAL HEALTH, CHILDREN, PRESCHOOL.

\section{INTRODUCCION}

En la infancia pueden ocurrir ciertos problemas bucales, tales como caries de infancia temprana, traumatismos dento-alveolares, maloclusiones y entre otros. En el Perú, estudios reportan una alta frecuencia de caries de infancia temprana oscilando entre el $11 \%$ al $96 \%(1,2)$, sin embargo en relación a las maloclusiones y traumatismos aún no existen estudios suficientes al respecto (3). Estos problemas pueden ocasionar repercusiones tales como dolor, dificultad para masticar, disminución del apetito, pérdida de peso, dificultad para dormir, alteración en el comportamiento, pérdida de días de clases en el colegio; por consiguiente la calidad de vida es afectada, además el niño no solamente se ve comprometido sino también los padres y el entorno familiar (4).

Para determinar los efectos directos e indirectos de los problemas de salud bucal en la calidad de vida se han desarrollado instrumentos en forma de cuestionarios, que complementan la información obtenida de la aplicación de indicadores clínicos tradicionales y son denominados indicadores sociodentales o de evaluación de la calidad de vida relacionado con la salud bucal-CVRSB $(5,6)$; de los que destaca Early Childhood Oral Health Impact Scale (ECOHIS), este ha sido diseñado por Pahel et al. para evaluar el impacto de los problemas dentales y/o experiencias de tratamientos odontológicos sobre la calidad de vida relacionada a la salud bucal de los niños menores de 5 años y sus familias, el cual ha sido adaptado transculturalmente y validado en diferentes países $(5,7,9)$.

Existen estudios sobre el impacto de los problemas bucales sobre la calidad de vida en niños menores de 5 años $(5,10,11)$ en relación al Perú no se conocen registros de estudios al respecto.

El propósito del presente estudio fue evaluar el impacto de los problemas bucales sobre la calidad de vida relacionada a la salud bucal en niños de 3 a 5 años de la Institución Educativa Inicial, del distrito de Hualmay, provincia de Huaura, departamento de Lima en el 2011.

\section{MATERIAL Y MÉTODOS}

El presente estudio de tipo descriptivo y transversal evaluó el impacto de los problemas bucales sobre la calidad de vida relacionada a la salud bucal en niños de 3 a 5 años de edad, asistentes a una Institución Educativa Inicial del distrito de Hualmay, provincia de Huaura, departamento de Lima. El cálculo del tamaño muestral se efectúo luego de la realización de la prueba piloto, se evaluaron 153 niños.

Los criterios de selección fueron: niños de ambos sexos y con padres o tutores bajo su responsabilidad, con aparente buen estado general de salud, con consentimiento informado de sus padres o tutores, y asentimiento informado y con actitud cooperadora.

Los métodos empleados fueron una encuesta estructurada y una observación directa. Las técnicas empleadas consistieron en: una aplicación de un cuestionario (ECOHIS) tipo entrevista dirigida al responsable del cuidado del menor y una evaluación clínica de los problemas bucales a los niños. Los datos generales del niño y del responsable del cuidado del menor fueron registrados en una ficha ad doc. En una prueba piloto se realizó la validación de la versión peruana del ECOHIS. El proceso de calibración interexaminador e intraexaminador fue realizado semanas previas a la recolección de datos, obteniendo índice de Kappa $\geq$ a 0,8.

La evaluación clínica de las alteraciones bucales se realizó de acuerdo a los criterios de la Organización Mundial de la salud (OMS)(22); en un ambiente proporcionado por el centro educativo; a través del método visual, bajo luz natural, con ayuda de un espejo bucal plano $\mathrm{N}^{\circ} 5$, baja lenguas y sondas periodontales, limpiando con gasas las superficies 
dentarias antes a ser examinadas y cumpliendo las normas de bioseguridad; se evaluaron las siguientes condiciones bucales:

Caries de Infancia Temprana (CIT): las lesiones fueron observadas en términos de dientes primarios cariados, extraídos y obturados. El índice ceo-d fue clasificado de acuerdo con la severidad de la CIT basados en los valores previamente descrito por Hallet et al.(23): $0=$ sin caries, ceo-d 1-5= baja gravedad y ceo- $d \geq 6=$ alta gravedad $(4,11)$.

Los traumatismos dento-alveolares fueron evaluados de acuerdo a la clasificación de Andreasen y Andreasen, 1994, el cual está basado en el sistema adoptada por el OMS, categorizados en ausencia y presencia $(4,15,11)$.

Las maloclusiones anteriores fueron evaluada según el tipo de maloclusión anterior: mordida abierta anterior (falta de contacto vertical entre los dientes incisivos del arco superior e inferior), entrecruzamiento horizontal (overjet $>3 \mathrm{~mm}$.), mordida profunda anterior, mordida cruzada anterior, mordida bis a bis $(4,12,11)$. Las medidas fueron realizadas con sondas periodontales según criterios OMS26 y fueron categorizadas por la presencia o ausencia de estas maloclusiones (4).

El Cuestionario ECOHIS: comprende 13 preguntas divididas en dos secciones una relacionada al Impacto en el niño (9 preguntas) y otra en relación al Impacto en la familia (4 preguntas). En donde el responsable del cuidado del menor selecciona la respuesta que mejor describe la experiencia de su hijo y la de él, considerando desde el nacimiento del menor hasta la actualidad. Teniendo como respuesta seis posibles opciones que son: nunca, casi nunca, ocasionalmente, a menudo, muy a menudo y una última opción no sabe (con valores equivalentes de 0 a 4 cada una). Luego se determina el resultado del cuestionario utilizando un método simple de conteo, para dar un total que varía de 0 a 52, en donde puntuaciones altas indican un impacto negativo o muchos problemas en la CVRSB. No se contabilizan aquellos cuestionarios que tenían dos o más preguntas sin responder en la sección relacionada al niño o una o más preguntas sin responder en la sección relacionada a la familia $(4,5)$.
Tanto las evaluaciones clínicas bucales a los niños y los cuestionarios dirigidos a los responsables del cuidado del menor se realizaron en un ambiente cedido por el centro educativo, estableciendo un cronograma para su realización.

En cuanto a las consideraciones éticas, se solicitó la aprobación del Comité de Ética Institucional de la Universidad Peruana Cayetano Heredia para la realización del presente estudio.

Los datos obtenidos fueron almacenados en una base de datos en el programa Excel y procesados en el programa estadístico SPSS versión 20.0. Se realizó el análisis estadístico: bivariado y bivariado, previa evaluación de la normalidad de los datos, comparándose las medias de la puntuación total del ECOHIS, los problemas bucales y las características de los niños y responsables de su cuidado entre sí; empleando pruebas paramétricas (Prueba Anova) y no paramétricas (Pruebas de U Mann Whitney y de Kruskal-Wallis) con un nivel de significancia de 95\%.

\section{RESULTADOS}

Entre las características observadas de los niños se halló que la edad de mayor frecuencia fue la edad de 5 años $(39,2 \%)$ y en relación al género el de mayor predominio fue el femenino $(54,9 \%)$. En cuanto a las características de los responsables del cuidado del menor se observaron que el sexo femenino fue el de mayor frecuencia con un $96,7 \%$ y la mayoría presentaba educación secundaria con un $75,8 \%$ (Tabla 1).

El $76,47 \%$ presentaban caries de la infancia temprana. El índice CEOD mostró un promedio de $3,57 \pm 3,32$. Los traumatismos dento-alveolares se presentaron en el $9,8 \%$ y con respecto a las maloclusiones anteriores se observaron en el $6,5 \%$ (Tabla 2).

Según la percepción del responsable sobre la CVRSB de su menor hijo, a través del ECOHIS, se encontró que las preguntas relacionadas al dolor $(54,9 \%)$, dificultades para comer $(49,7 \%)$, manifestación de enojado o frustración $(48,4 \%)$ y sobre si el responsable del niño u otro familiar ha estado alterado $(47,1 \%)$ fueron las de mayor frecuencia (Tabla 3). 
Tabla 1. Características de los niños y de los responsables del cuidado del menor, del Centro Educativo Inicial del distrito de Hualmay, provincia de Huaura, Lima, 2011 ( $n=153)$.

\begin{tabular}{|c|c|c|}
\hline $\begin{array}{l}\text { CARACTERÍSTICAS DE LOS NIÑOS } \\
\text { Y DE LOS RESPONSABLES DE } \\
\text { CUIDADO DEL MENOR }\end{array}$ & $\mathrm{n}$ & $\%$ \\
\hline \multicolumn{3}{|l|}{ EDAD } \\
\hline 3 años & 47 & 30,72 \\
\hline 4 años & 46 & 30,07 \\
\hline 5 años & 60 & 39,22 \\
\hline \multicolumn{3}{|l|}{ GENERO } \\
\hline Masculino & 69 & 45,10 \\
\hline Femenino & 84 & 54,90 \\
\hline \multicolumn{3}{|l|}{$\begin{array}{l}\text { GENERO DEL RESPONSABLE DEL } \\
\text { CUIDADO DEL MENOR }\end{array}$} \\
\hline Masculino & 5 & 3,27 \\
\hline Femenino & 148 & 96,73 \\
\hline \multicolumn{3}{|l|}{$\begin{array}{l}\text { EDAD DEL RESPONSABLE DEL } \\
\text { CUIDADO DEL MENOR }\end{array}$} \\
\hline 15 - 30 años & 85 & 55,56 \\
\hline 31 - 46 años & 59 & 38,56 \\
\hline 47 - 62 años & 9 & 5,88 \\
\hline \multicolumn{3}{|l|}{$\begin{array}{l}\text { NIVEL DE INSTRUCCIÓN DEL } \\
\text { RESPONSABLE DEL CUIDADO DEL } \\
\text { MENOR }\end{array}$} \\
\hline Primaria & 7 & 4,58 \\
\hline Secundaria & 116 & 75,82 \\
\hline Técnica & 9 & 5,88 \\
\hline Universitaria & 21 & 13,73 \\
\hline \multicolumn{3}{|l|}{$\begin{array}{l}\text { PARENTESCO DEL RESPONSABLE } \\
\text { DEL CUIDADO DEL MENOR CON EL } \\
\text { NIÑO }\end{array}$} \\
\hline Madre & 138 & 90,20 \\
\hline Padre & 3 & 1,96 \\
\hline Otro & 12 & 7,84 \\
\hline
\end{tabular}

La puntuación total máxima del ECOHIS fue 46 y la mínima fue 0 , siendo el promedio $15,50 \pm 10,24$. Presentando la dimensión limitación funcional el promedio más alto 4,75 (Tabla 4).

La comparación de los promedios entre las características de los niños y de los responsables del menor con respecto al promedio de la puntuación total del ECOHIS no se encontraron asociación, tampoco se observó por las dimensiones del mismo con un p>.05 (Tabla 5).
Tabla 2. Frecuencia de los problemas bucales en niños de 3 a 5 años del Centro Educativo Inicial del distrito de Hualmay, provincia de Huaura, departamento de Lima, $2011(n=153)$.

\begin{tabular}{lcc}
\hline \multicolumn{1}{c}{ PROBLEMAS BUCALES } & $\mathrm{n}$ & $\%$ \\
\hline CARIES DE INFANCIA & & \\
TEMPRANA & 36 & 23,53 \\
Sanos & 77 & 50,33 \\
Severidad Baja & 40 & 26,14 \\
Severidad Alta & & \\
TRAUMATISMOS DENTO- & & \\
ALVEOLARES & 138 & 90,20 \\
Ausencia & 15 & 9,80 \\
Presencia & & \\
MALOCLUSIONES ANTERIORES & 143 & 93,46 \\
Ausencia & 10 & 6,54 \\
Presencia &
\end{tabular}

Mientras las comparaciones entre las medias de la puntuación total del ECOHIS, por sus dimensiones, y las condiciones bucales; se encontró que la caries de infancia temprana presentó un promedio elevado con un $24,88 \pm 9,43$ (Tabla 6).

\section{DISCUSIÓN}

El presente estudio evaluó el impacto de los problemas bucales como caries de infancia temprana, traumatismos dento-alveolares y las maloclusiones anteriores sobre la calidad de vida relacionada a la salud bucal en 153 niños en edad preescolar.

La caries de infancia temprana fue hallada con una alta frecuencia $(76,47 \%)$. Así también lo reportan en forma semejante estudios anteriores $(5,9,10,11)$. Sin embargo, Wong y col. y Kramer et al., reportaron una prevalencia de caries de infancia temprana menor. La diversidad de resultados obtenidos podría ser explicada por la cantidad de niños examinados, el rango de edades, la situación del contexto social y geográfico en que se realizaron los estudios y así como también por el indicador clínico empleado en la evaluación de caries de infancia temprana.

Los traumatismos dento-alveolares se presentaron en menor frecuencia $(9,8 \%)$. Otras investigaciones encontraron una frecuencia relativamente mayor de 
Tabla 3. Percepción del responsable del cuidado del menor sobre calidad de vida relacionada a la salud bucal del Centro Educativo Inicial, distrito de Hualmay, provincia de Huaura, departamento de Lima, 2011 ( $n=153$ ).

\begin{tabular}{|c|c|c|c|}
\hline \multirow[t]{2}{*}{$\begin{array}{l}\text { ESCALA DE IMPACTO EN LA SALUD BUCAL } \\
\text { EN LA INFANCIA TEMPRANA- ECOHIS }\end{array}$} & $\begin{array}{c}\text { Nunca/ Casi } \\
\text { nunca }\end{array}$ & $\begin{array}{c}\text { Ocasionalmente/ A } \\
\text { menudo/ Muy a menudo }\end{array}$ & No sabe \\
\hline & $\mathrm{n}(\%)$ & $\mathrm{n}(\%)$ & $\mathrm{n}(\%)$ \\
\hline \multicolumn{4}{|l|}{ IMPACTO EN EL NIÑO } \\
\hline 1. Dolor en los dientes, boca o mandíbula & $68(44,4)$ & $84(54,9)$ & $1(0,7)$ \\
\hline 2. Dificultades para tomar bebidas calientes o frías & $88(57,5)$ & $64(41,9)$ & $1(0,7)$ \\
\hline 3. Dificultades para comer algunos alimentos & $77(50,3)$ & $76(49,7)$ & $0(0,0)$ \\
\hline 4. Dificultades para pronunciar algunas palabras & $107(69,9)$ & $44(28,8)$ & $2(1,3)$ \\
\hline $\begin{array}{l}\text { 5. Perdida de días de asistencia a su actividad } \\
\text { preescolar, guardería o escuela. }\end{array}$ & $113(73,8)$ & $40(26,2)$ & $0(0,0)$ \\
\hline 6 Problemas para dormir & $102(66,7)$ & $51(33,4)$ & $0(0,0)$ \\
\hline 7. Ha estado enojado o frustrado & $79(51,6)$ & $74(48,4)$ & $0(0,0)$ \\
\hline 8. Ha evitado sonreír & $119(77,8)$ & $33(21,6)$ & $1(0,7)$ \\
\hline 9. Ha evitado hablar & $121(79,1)$ & $32(20,9)$ & $0(0,0)$ \\
\hline \multicolumn{4}{|l|}{ IMPACTO EN LA FAMILIA } \\
\hline $\begin{array}{l}\text { 10. Usted u otro miembro de la familia ha estado } \\
\text { preocupado }\end{array}$ & $81(52,9)$ & $72(47,1)$ & $0(0,0)$ \\
\hline $\begin{array}{l}\text { 11. Usted u otro miembro de la familia se ha } \\
\text { sentido culpable }\end{array}$ & $94(61,4)$ & $59(38,5)$ & $0(0,0)$ \\
\hline $\begin{array}{l}\text { 12. Usted u otro miembro de la familia ha ocupa- } \\
\text { do tiempo. }\end{array}$ & $98(64,1)$ & $55(36,0)$ & $0(0,0)$ \\
\hline 13.Han tenido un impacto económico en su familia & $94(61,4)$ & $59(38,6)$ & $0(0,0)$ \\
\hline
\end{tabular}

estas condiciones $(5,12,13)$. Por el contrario Viegas et al y Scarpelli et al. hallaron una prevalencia de traumatismos alta. Según Abanto et al, la evaluación visual que aplicaron en los estudios puede subestimar la presencia de traumatismos dento-alveolares; ya que no se emplearon exámenes radiográficos (4).

Las maloclusiones anteriores fueron halladas en menor frecuencia, similares a estudios anteriores $(4,12)$. Abanto et al. mencionan que estos resultados pueden ser debido a que solamente fueron consideradas las maloclusiones anteriores, por presentar mayor compromiso estético; ya que no existe aún una clasificación de maloclusión disponible para niños menores de 5 años (4). Sin embargo, Scarpelli et al. y Kramer et al., reportaron una frecuencia mayor de maloclusiones, debido posiblemente al tamaño muestral estudiado en donde los hallazgos tienden a ser mayores.

Las preguntas respondidas por los responsables del cuidado del menor con mayor predomino, en el
ECOHIS; fueron las relacionadas al dolor, dificultades para comer, enojo o frustración. Otros estudios reportan similares resultados $(4,12,14,11,13)$, por otra parte, coincidiendo con Abanto et al, los padres respondieron más la sección relacionada al niño que de la familia (4). Esto podría deberse a que las enfermedades y los transtornos bucales al ser más graves, la relación padre-hijo es un poco más cercana cuando los niños han presentado estas condiciones; por lo que los padres están más familiarizados con sus actividades y sentimientos $(4,5,10)$.

La caries de infancia temprana presentó un impacto negativo con la CVRSB, similares hallazgos fueron encontrados en otros estudios $(4,12,14,15,11,16)$. Abanto et al. Observaron que el incremento en la severidad de caries dental en los niños presentó impacto negativo en la calidad de vida según la percepción de los padres, y esto es esperado debido al hecho que la enfermedad puede originar estas emociones, ya que la severidad de la caries usualmente produce una apariencia desagradable; el cual podría 
Tabla 4. Evaluación de la Calidad de vida relacionada a la salud bucal según el impacto en el niño y en la familia del Centro Educativo Inicial, distrito de Hualmay, provincia de Huaura, departamento de Lima, 2011 (n=153).

\begin{tabular}{|c|c|c|c|c|}
\hline \multirow[t]{2}{*}{$\begin{array}{c}\text { ESCALA DE IMPACTO EN LA SALUD } \\
\text { BUCAL EN LA INFANCIA TEMPRANA- } \\
\text { ECOHIS }\end{array}$} & \multicolumn{4}{|c|}{$\begin{array}{c}\text { CALIDAD DE VIDA RELACIONADA A LA SALUD } \\
\text { BUCAL } \\
\text { ECOHIS }\end{array}$} \\
\hline & MEDIA & $\begin{array}{c}\text { DESVIACIÓN } \\
\text { ESTÁNDAR }\end{array}$ & MÍNIMO & MÁXIMO \\
\hline \multicolumn{5}{|l|}{ SECCIÓN IMPACTO EN EL NIÑO } \\
\hline Síntomas Orales (1) & 1,54 & 1,03 & 0 & 4 \\
\hline Limitaciones Funcionales (4) & 4,75 & 3,44 & 0 & 13 \\
\hline Aspectos Psicológicos (2) & 2,31 & 1,79 & 0 & 7 \\
\hline Autoimagen/interacción social (2) & 1,74 & 1,63 & 0 & 7 \\
\hline \multicolumn{5}{|l|}{ SECCIÓN IMPACTO EN LA FAMILIA } \\
\hline Angustia de los padres (2) & 2,71 & 1,90 & 0 & 8 \\
\hline Función Familiar (2) & 2,46 & 1,75 & 0 & 8 \\
\hline PUNTUACIÓN TOTAL ECOHIS & 15,50 & 10,24 & 0 & 46 \\
\hline
\end{tabular}

( ) Cantidad de preguntas

preocupar a los padres en relación a las oportunidades sobre la vida futura del niño (15).

Por otro lado, se observó un impacto negativo en las dimensiones del ECOHIS, siendo la dimensión de autoimagen/interacción social la de menor frecuencia de respuesta; así también lo reportaron Abanto y col (4). Hetherington menciona que de acuerdo con la psicología del desarrollo del niño, a la edad de seis años es el comienzo del pensamiento abstracto, la auto-imagen y el concepto (24). Esto es también explicado por el hecho de que la investigación evaluó niños menores de 6 años de edad. Esto podría ser una de las razones de la baja frecuencia de respuestas en esta dimensión (4). Además, la dimensión angustia de los padres/ cuidadores presentó mayor predominio debido a que los niños presentaron problemas bucales; otros estudios reportan semejantes resultados $(4,15)$. Por otro lado, Anderson y col. Han indicado que la caries de infancia temprana resulta en días perdidos de trabajo para los cuidadores que tienen que quedarse en casa para cuidar de su hijo o tienen que gastar tiempo y dinero en la atención dental (15). Abanto et al., afirman que estas influencias sobre los padres o cuidadores son importantes para medir, como parte de la evaluación de CVRSB de los niños menores (4). En la actualidad, existe una fuerte evidencia que los padres o los cuidadores de niños pequeños experimentan de manera significativa los temas sobre calidad de vida porque son problemas de salud y experiencias de tratamientos en sus hijos $(4,12,15)$.

Los traumatismos dento-alveolares y las maloclusiones anteriores no se asociaron con un impacto negativo en CVRSB. Abanto et al. y Scarpelli et al., encontraron similares resultados $(4,11)$. Kramer et al., mencionan que las limitaciones en algunas de las funciones, usualmente, son observadas en las complicaciones de los traumatismos (11), además Aldrigui y col. afirman que según la complicación del traumatismo dento-alveolar sí tiene un impacto negativo en la CVRSB (12); puesto que la complicación del traumatismo, con exposición de tejido pulpar, puede demandar mayor tiempo a los miembros de la familia a tener en cuenta los síntomas producidos por los traumatismos (12). Además, el no hallar una asociación con la CVRSB es probablemente debido al hecho de que la mayor parte de traumatismos encontrados en los niños fueron lesiones de los tejidos del esmalte dental, que son lesiones leves. Sin embargo, existe limitada evidencia en la actualidad sobre esta asociación $(4,12)$.

En relación a las maloclusiones anteriores no se asociaron con un impacto negativo en la CVRSB de los niños y sus padres, esto es semejante con otros estudios $(4,12,11,16)$. Abanto menciona que de manera diferente a los traumatismos, que suceden 
Tabla 5. Evaluación de las características de los niños y del responsable del cuidado del menor sobre la calidad de vida relacionada a la salud bucal del Centro Educativo Inicial, distrito de Hualmay, provincia de Huaura, departamento de Lima, 2011 ( $\mathrm{n}=153)$.

\begin{tabular}{|c|c|c|c|}
\hline \multirow{2}{*}{$\begin{array}{c}\text { CARACTERÍSTICAS DEL NIÑO Y DEL RESPONSABLE } \\
\text { DEL CUIDADO DEL MENOR }\end{array}$} & \multicolumn{3}{|c|}{$\begin{array}{l}\text { CALIDAD DE VIDA RELACIONADA A LA SALUD } \\
\text { BUCAL } \\
\text { ECOHIS }\end{array}$} \\
\hline & MEDIA & $\begin{array}{l}\text { DESVIACIÓN } \\
\text { ESTÁNDAR }\end{array}$ & VALOR p \\
\hline \multicolumn{4}{|l|}{ EDAD DEL NIÑO } \\
\hline 3 años & 14,62 & 9,88 & \\
\hline 4 años & 14,41 & 11,25 & \\
\hline 5 años & 17,02 & 9,68 & $0,338 \mathrm{a}$ \\
\hline \multicolumn{4}{|l|}{ SEXO DEL NIÑO } \\
\hline Masculino & 15,46 & 10,98 & \\
\hline Femenino & 15,52 & 9,66 & $0,772 b$ \\
\hline \multicolumn{4}{|l|}{ SEXO DEL RESPONSABLE DEL MENOR } \\
\hline Masculino & 15,00 & 15,75 & \\
\hline Femenino & 15,51 & 10,09 & $0,590 \mathrm{~b}$ \\
\hline \multicolumn{4}{|l|}{ EDAD DEL RESPONSABLE DEL MENOR } \\
\hline 15 - 30 años & 15,56 & 10,01 & \\
\hline 31 - 46 años & 15,54 & 10,42 & \\
\hline 47 - 62 años & 14,56 & 12,39 & $0,961 \mathrm{a}$ \\
\hline \multicolumn{4}{|l|}{$\begin{array}{l}\text { NIVEL DE INSTRUCCIÓN DEL RESPONSABLE DEL } \\
\text { MENOR }\end{array}$} \\
\hline Primaria & 18,43 & 9,54 & \\
\hline Secundaria & 15,84 & 10,55 & \\
\hline Técnica & 14,00 & 10,44 & \\
\hline Universitaria & 13,29 & 8,71 & $0,604 \mathrm{a}$ \\
\hline \multicolumn{4}{|l|}{ PARENTESCO DEL RESPONSABLE DEL MENOR } \\
\hline Madre & 15,72 & 10,23 & \\
\hline Padre & 7,33 & 8,08 & \\
\hline Otro & 15,00 & 10,72 & $0,325 \mathrm{c}$ \\
\hline \multicolumn{4}{|l|}{ aPrueba Anova } \\
\hline \multicolumn{4}{|l|}{ bPrueba U de Mann Whitney } \\
\hline cPrueba Kruskal Wallis & & & \\
\hline
\end{tabular}

repentinamente, en las maloclusiones los cambios en la dentición se producen lentamente y en las etapas de desarrollo (4). Por lo tanto, las maloclusiones por lo general pasan desapercibidas en los niños y los padres y la mayoría de ellos no saben las consecuencias estéticas, psicológicas y económicas que pueden producir estas maloclusiones en edades más avanzadas(4).
En el presente estudio, solamente, la caries de infancia temprana presentó impacto negativo sobre la calidad de vida relacionada a la salud bucal en los niños menores de 5 años y sus familias. Además, se recomienda realizar estudios futuros en relación a esta temática, puesto que brinda información a las entidades tanto públicas como privadas, a los profesionales de salud y población en general de los 
Tabla 6. Evaluación de los Problemas Bucales sobre la calidad de vida relacionada a la salud bucal de niños de 3 a 5 años del Centro Educativo Inicial, distrito de Hualmay, provincia de Huaura, departamento de Lima, 2011 ( $\mathrm{n}=153$ ).

\begin{tabular}{|c|c|c|c|c|c|c|c|c|}
\hline \multirow[b]{2}{*}{ PROBLEMAS BUCALES } & \multirow[b]{2}{*}{$\mathrm{n}(\%)$} & \multicolumn{7}{|c|}{$\begin{array}{l}\text { CALIDAD DE VIDA RELCIONADA A LA SALUD BUCAL- ECOHIS } \\
\text { DIMENSIONES }\end{array}$} \\
\hline & & $\begin{array}{l}\text { Síntomas } \\
\text { Orales }\end{array}$ & $\begin{array}{l}\text { Limitación } \\
\text { Funcional }\end{array}$ & $\begin{array}{l}\text { Aspectos } \\
\text { Psicológicos }\end{array}$ & $\begin{array}{l}\text { Autoimagen/ } \\
\text { Interacción } \\
\text { Social }\end{array}$ & $\begin{array}{l}\text { Angustia de } \\
\text { los Padres }\end{array}$ & $\begin{array}{l}\text { Función } \\
\text { Familiar }\end{array}$ & $\begin{array}{c}\text { PUNTUACIÓN } \\
\text { TOTAL } \\
\text { ECOHIS }\end{array}$ \\
\hline \multicolumn{9}{|l|}{ CARIES DE LA INFANCIA TEMPRANA } \\
\hline Sano & $36(23,53)$ & $0,92(0,77)$ & $2.78(2.74)$ & $0,23(1,35)$ & $1,22(1,22)$ & $1,42(1,18)$ & $0,21(1,20)$ & $8,75(7,33)$ \\
\hline Severidad Baja & $77(50,33)$ & $1,35(0,87)$ & $4,07(2,83)$ & $2,05(1,47)$ & $1,56(1,44)$ & $2,52(1,68)$ & $2,24(1,64)$ & $13,78(8,28)$ \\
\hline Severidad Alta & $40(26,14)$ & $2,45(0,90)$ & $7,83(3,10)$ & $3,98(1,46)$ & $2,55(2,00)$ & $4,25(1,82)$ & $3,83(1,53)$ & $24,88(9,43)$ \\
\hline Valor $\mathrm{p}$ & & $\mathrm{p}<.001 \mathrm{c}$ & $\mathrm{p}<.001 \mathrm{c}$ & $\mathrm{p}<.001 \mathrm{c}$ & $0,003 \mathrm{c}$ & $\mathrm{p}<.001 \mathrm{c}$ & $\mathrm{p}<.001 \mathrm{c}$ & $\mathrm{p}<.001 \mathrm{a}$ \\
\hline \multicolumn{9}{|c|}{ TRAUMATISMOS DENTO-ALVEOLARES } \\
\hline Ausencia & $138(90,20)$ & $1,51(1,05)$ & $4,66(3,48)$ & $2,26(1,81)$ & $1,73(1,68)$ & $2,65(1,91)$ & $2,42(1,80)$ & $15,22(10,42)$ \\
\hline Presencia & $15(9,80)$ & $1,80(0,78)$ & $5,53(3,00)$ & $2,73(1,62)$ & $1,87(1,19)$ & $3,27(1,83)$ & $2,87(1,19)$ & $18,07(8,22)$ \\
\hline Valor $\mathrm{p}$ & & $0,235 b$ & $0,325 b$ & $0,227 \mathrm{~b}$ & $0,486 \mathrm{~b}$ & $0,220 \mathrm{~b}$ & $0,174 \mathrm{~b}$ & $0,187 \mathrm{~b}$ \\
\hline \multicolumn{9}{|l|}{ MALOCLUSIONES ANTERIORES } \\
\hline Ausencia & $143(93,46)$ & $1,52(1,03)$ & $4,69(3,42)$ & $2,27(1,77)$ & $1,68(1,50)$ & $2,65(1,83)$ & $2,41(1,73)$ & $15,21(9,97)$ \\
\hline Presencia & $10(6,54)$ & $1,80(1,03)$ & $5,50(3,81)$ & $2,90(2,13)$ & $2,60(2,95)$ & $3,60(2,76)$ & $3,20(1,93)$ & $19,6(13,51)$ \\
\hline Valor $\mathrm{p}$ & & $0,471 \mathrm{~b}$ & $0,558 \mathrm{~b}$ & $0,360 \mathrm{~b}$ & $0,546 \mathrm{~b}$ & $0,294 b$ & $0,186 b$ & $0,335 b$ \\
\hline
\end{tabular}

Media (Desviación estándar)

aPrueba Anova

bPrueba U de Mann- Whitney

cPrueba Kruskal- Wallis

efectos de los problemas bucales sobre la calidad de vida del niño y de su familia; de esta manera se podrán implementar programas de promoción y prevención en salud bucal realmente eficaces y oportunas.

\section{CORRESPONDENCIA}

Roxana Patricia López Ramos

Jirón Yungay 1663. Lima 01, Perú

Teléfono: 425-5696/945753623

e-mail: roxi_lpz@hotmail.com

\section{REFERENCIAS BIBLIOGRÁFICAS}

1. Bernabé E, Delgado E, Sánchez P. Resultados de un sistema para la vigilancia de la caries de la infancia temprana. Rev Med Hered. 2006;17(4):227-33.

2. Villena R, Pachas F, Sánchez Y, Carrasco M. Prevalencia de caries de infancia temprana en niños menores de 6 años de edad, residentes en poblados urbano marginales de Lima Norte. Rev Estomatol Herediana. 2011;21(2):79-86.

3. Williams F. Valverde R, Meneses A. Dimensiones de $\operatorname{arcos}$ y relaciones oclusales en dentición decidua completa. Rev Estomatol Herediana. 2004;14(1-2):2246.

4. Abanto J, Carvalho T, Mendes F, Wanderley M, Bönecker $\mathrm{M}$, Raggio D. Impact of oral diseases and disorders on oral health- related quality of life of preschool chil-dren. Community Dent Oral Epidemiol. 2011 Apr;39(2):105-
14.

5. Pahel B, Rozier R, Slade G. Parental perceptions of children's oral health: the Early Childhood Oral Health Impact Scale. Health Qual Life Outcomes. 2007;30(5):6.

6. Tesch F, Oliveira B, Leão A. Semantic equivalence of the Brazilian version of the Early Childhood Oral Health Impact Scale. Cad Saude Publica. 2008 Aug;24(8):1897909.

7. Li S. Veronneau J, Allison P. Validation of a French Language version of the Early Childhood Oral Health Impact Scale. Health Qual Life Outcomes. 2008 Jan 22;6:9.

8. Lee G, McGrath C, Yiu C, King N. Translation and validation of a Chinese language version of the Early Childhood Oral Health Impact Scale (ECOHIS). Int J Paediatr Dent. 2009 Nov;19(6):399-405.

9. Bordoni N, Ciaravino O, Zambrano O, Villena R, Beltran-Aguilar E, Squassi A. Early Childhood Oral Health Impact Scale (ECOHIS). Translation and validation in Spanish language. Acta Odontol Latinoam. 2012;25(3):270-8.

10.Gradella CM, Bernabé, Bonecker M, Oliveira LB. Caries prevalence and severity, and quality of life in Brazilian 2-to 4-year-oldchildren. Community Dent Oral Epi-demiol. 2011;39:498-504.

11.Kramer PF, Feldens CA, Helena Ferreira S, Bervian J, Rodrigues PH, Peres MA. Exploring the impact of oral diseases and disorders on quality of life of preschool children. Community Dent Oral Epidemiol. 2013 Aug;41(4):327-35.

12.Aldrigui J, Abanto J, Carvalho T, Mendes F, Wanderley M, Bönecker M, Raggio D. Impact of traumatic dental 
injuries and malocclusions on quality of life of young children. Health Qual Life Outcomes. 2011 Sep 24;9:(78):1-7.

13. Organización Mundial de la Salud. Encuestas de la salud bucodental: métodos bá-sicos.4ta ed. Ginebra: Organización Mundial de la Salud; 1997.

14. Wong HM, McGrath CP, King NM, Lo EC. Oral health- related of life in Hong Kong preschool children. Caries Res 2011;45:370-6.

15.Leal S, Bronkhorst E, Fan M, Frencken J. Untreated Cavitated Dentine Lesions: Impact on Children's Quality of Life. Caries Res. 2012 Mar 6;46(2):102-6.

16. Hallet K, O'rourke P. Pattern and severity of early childhood caries. Community Dent Oral Epidemiol. 2006 Feb;34(1):25-35.

Recibido : 06-09-2012

Aceptado: 24-12-2012 